\title{
Defective chromatic and achromatic
} visual pathways in developmental

\section{dyslexia: Cues for an integrated intervention programme}

\author{
Luca Bonfiglio $^{\mathrm{a}, *}$, Tommaso Bocci ${ }^{\mathrm{b}}$, Fabrizio Minichillic ${ }^{\mathrm{c}}$, Alessandra Crecchi ${ }^{\mathrm{a}}$, \\ Davide Barloscio ${ }^{\mathrm{b}}$, Donata Maria Spina ${ }^{\mathrm{d}}$, Bruno Rossi ${ }^{\mathrm{a}}$ and Ferdinando Sartucci ${ }^{\mathrm{b}}$ \\ ${ }^{a}$ Department of Translational Research on New Technologies in Medicine and Surgery, \\ School of Physical Medicine and Rehabilitation, University of Pisa, Pisa, Italy \\ ${ }^{\mathrm{b}}$ Department of Clinical and Experimental Medicine, Cisanello Neurology Unit, Pisa University \\ Medical School, Pisa, Italy \\ ${ }^{\mathrm{c}}$ Unit of Environmental Epidemiology, Institute of Clinical Physiology, National Council of Research, \\ Pisa, Italy \\ ${ }^{\mathrm{d}}$ Children's Neuropsychiatric Medical Facility, Local Health Authority of Viareggio (USL 12), \\ Lido di Camaiore (LU), Italy
}

\begin{abstract}
.
Purpose: As well as obtaining confirmation of the magnocellular system involvement in Developmental dyslexia (DD); the aim was primarily to search for a possible involvement of the parvocellular system; and, furthermore, to complete the assessment of the visual chromatic axis by also analysing the koniocellular system.

Methods: Visual evoked potentials (VEPs) in response to achromatic stimuli with low luminance contrast and low spatial frequency, and isoluminant red/green and blue/yellow stimuli with high spatial frequency were recorded in 10 dyslexic children and 10 age- and sex-matched, healthy subjects.

Results: Dyslexic children showed delayed VEPs to both achromatic stimuli (magnocellular-dorsal stream) and isoluminant $\mathrm{red} /$ green and blue/yellow stimuli (parvocellular-ventral and koniocellular streams). To our knowledge, this is the first time that a dysfunction of colour vision has been brought to light in an objective way (i.e., by means of electrophysiological methods) in children with DD.

Conclusion: These results give rise to speculation concerning the need for a putative approach for promoting both learning how to read and/or improving existing reading skills of children with or at risk of DD. The working hypothesis would be to combine two integrated interventions in a single programme aimed at fostering the function of both the magnocellular and the parvocellular streams.
\end{abstract}

Keywords: Reading disorder, letter recognition, parvocellular, magnocellular, koniocellular, chromatic contrast, luminance contrast, VEPs

\section{Introduction}

${ }^{*}$ Corresponding author: Luca Bonfiglio, Department of Translational Research on New Technologies in Medicine and Surgery, School of Physical Medicine and Rehabilitation, University of Pisa, Via Roma 67, I-56126 Pisa, Italy. Tel./Fax: +39050 992655; E-mails: 1.bonfiglio@ao-pisa.toscana.it; bubobis @gmail.com.
The human visual system consists of three parallel pathways that originate from different retinal ganglion cells and, after making a relay on specific areas 
of the lateral geniculate nucleus, converge on visual cortical areas V1 and V2: a) the magnocellular pathway, which originates from the parasol ganglion cells and projects onto large magnocellular neurons of the dorsal lateral geniculate nucleus; b) the parvocellular pathway, which originates from the midget ganglion cells and projects onto small parvocellular neurons of the ventral lateral geniculate nucleus; c) the koniocellular pathway, which originates from the bistratified ganglion cells and projects onto the neurons of the intercalated layers of the lateral geniculate nucleus (Chatterjee \& Callaway, 2002; Sumner et al., 2008; Ribeiro \& Castelo-Branco, 2010).

Beyond the early visual cortical areas, the two main systems form two segregated projective pathways towards extrastriate and associative visual cortical areas: magnocellular inputs are conveyed to V5/MT and the posterior parietal cortex (magnocellulardorsal stream) (Born \& Bradley, 2005), while parvocellular inputs to $\mathrm{V} 4$ and the inferotemporal cortex (parvocellular-ventral stream) (Heywood et al., 1992).

The magnocellular system, being composed of large neurons characterised by high excitability and conduction velocity, is a phasic system capable of producing rapid and short responses. It is mainly sensitive to object movements and achromatic stimuli with low spatial frequency, high temporal frequency and low luminance contrast. It is basically concerned with parafoveal and peripheral vision and its main physiological function is to direct attention to the spatial characteristics (localisation) of the object (where pathway) (Vidyasagar, 1999; Laycock et al., 2008; Brown, 2009).

The parvocellular system, however, being composed of small neurons characterised by lower excitability and conduction velocity, is a tonic system capable of producing late responses, which are however more sustained over time. It is mainly sensitive to isoluminant red/green chromatic stimuli with high spatial frequency, low temporal frequency and high chromatic contrast. It is basically concerned with foveal or central vision and its main physiological function is to focus attention on the proper characteristics (recognition) of the object (what pathway) (Vidyasagar, 1999; Laycock et al., 2008; Brown, 2009).

The koniocellular system is mainly sensitive to isoluminant blue/yellow chromatic stimuli, but also contributes to object movement perception through projections onto V5/MT, which is part of the magnocellular-dorsal stream (Shipp, 2006).

\subsection{Developmental dyslexia}

Developmental Dyslexia (DD) consists of a deficit in acquiring adequate reading skills and occurs despite the lack of any neurological, cognitive, sensorial and social disability in subjects with normal intelligence and normal educational opportunities (Lyon et al., 2003; Peterson \& Pennington, 2012).

At present, the main problem of DD is believed to depend on an impaired processing of auditory and phonological stimuli (phonological awareness theory) (Peterson \& Pennington, 2012; Hornickel \& Kraus, 2013), however, several theories have been called into play over the years to explain the physiopathogenesis of DD, some of which still retain their validity (for review see Paulesu et al., 2014).

One of them is the magnocellular theory, which is based on a perceptual defect of the magnocellular system (Lovegrove et al., 1982; Galaburda \& Livingstone, 1993). The original assumption of this theory was based on the belief that, in normal reading, the parvocellular system is active during fixations, as opposed to the magnocellular system which is active during saccades. In this way, the magnocellular stream would seemingly exert an inhibitory effect on the parvocellular system only during saccades, thus preventing parvocellular neuronal activity from continuing until the next fixation. In DD, however, given the impaired inhibition of the parvocellular system due to the magnocellular weakness, an overlay of images arising from two subsequent fixations would appear to occur, with the final effect of confusing the reading.

This hypothesis, however, has already been called into question by Burr and colleagues (Burr et al., 1994), who have shown that during saccades the magnocellular system actually inhibits its own previous activity, rather than that of the parvocellular system, thus confuting the intimate mechanism from which the reading impairment was thought to arise. Nevertheless, this finding was not incompatible with the possibility that a deficit of the magnocellular system contributes, to some extent, to the neurobiological substrate of DD. Since the early 90s, in fact, several confirmations of a magnocellular dysfunction have been recorded for children with dyslexia: abnormal responses have been reported for those characteristics of visual stimuli that are specifically targeted by the magnocellular system, namely, achromatic vision, low spatial frequency, low contrast (May et al., 1991; Maddock et al., 1992; Romani et al., 2001; Samar et al., 2002; Vaegan \& Hollows, 2006), 
high temporal frequency and object movement perception (Kubová et al., 1996; Kuba et al., 2001; Schulte-Körne et al., 2004). These findings fit with anatomo-structural observations that magnocellular neurons of the lateral geniculate nucleus of dyslexic children are both smaller and dystrophic compared with those of age-matched normal readers (Livingstone et al, 1991). Recently, a reduced activation of V5/MT during visual processing of moving objects has been demonstrated in dyslexic children (Olulade et al., 2013). However, no activation deficit has emerged with respect to younger children matched for reading skills (i.e., with the same reading skills), suggesting that the magnocellular dysfunction can be considered an effect rather than the cause of DD. At present, the magnocellular dysfunction is considered to be connected to an impairment of visual attentional shifting, both in its spatial and temporal aspects (Hari \& Renvall, 2001). Indeed, this is a crucial skill in the segmentation of letter strings into grapheme constituents (graphemic parsing) (Gori \& Facoetti, 2014) and is, therefore, a preliminary condition to the letter-to-speech sound integration.

As far as the parvocellular system is concerned, for many years it has been investigated using visual stimuli with high spatial-frequency (i.e., within the characteristic sensitivity range of the parvocellular system) but achromatic (i.e., stimuli towards which the parvocellular system is substantially blind, whilst the magnocellular system is maximally sensitive) (Victor et al., 1998). In this way, a unique electrophysiological study was able to demonstrate delayed responses in dyslexic children compared to controls (Farrag et al., 2002), although the lack of selectivity of the visual stimuli employed did not exclude the possibility of a contribution from the magnocellular system to the abnormal responses. On the other hand, more recently Ahmadi et al. (2015) have been able to demonstrate an impairment of the paryocellular system in dyslexic children. They did this by presenting coloured visual stimuli (i.e., highly selective for the parvocellular system) in the form of images of natural scenes and by determining the red-green isoluminant point using the psychophysical (subjective) method.

\subsection{Aims and scope}

In the present study we used an electrophysiological (objective) method. This is represented by visual evoked potentials (VEPs), capable of disclosing even subtle functional impairments of the investigated systems. This is more sensitive even than the selfawareness that tested subjects may have of their own dysfunctions (for rev. see Tobimatsu \& Celesia, 2006).

The primary aims of the present study were: a) to obtain a confirmation of the magnocellular system involvement, by using achromatic stimuli with low luminance contrast and low spatial frequency; b) to search for any potential involvement of the parvocellular system, by using isoluminant red/green stimuli with high spatial frequency, to which this system is specifically sensitive, (i.e. the most suitable stimuli for selectively stimulating this system).

An additional aim was c) to complete the functional assessment of the visual chromatic axis by using isoluminant blue/yellow chromatic stimuli in order to analyse also the koniocellular system.

\section{Materials and methods}

\subsection{Participants}

Ten dyslexic children (5 girls; mean age $142,3 \pm 14,3$ months) were selected from a sample of children referred to the Children's Neuropsychiatric Medical Facility of Viareggio (Italy). These children had been diagnosed as dyslexic by an expert paediatric neuropsychiatrist (D.M.S.) on the basis of the Italian National Recommendations (VV.AA.). These require that the child have (a) a full-scale IQ within the normal range (i.e., greater than 85), as measured by the Wechsler Intelligence Scale for Children (3rd edition; WISC-III) (Orsini \& Picone, 2006), and (b) a performance two negative SDs below age group norms in one reading task, or one negative SD in at least two reading tasks of the standard Italian test for assessment of reading skills. This test consists of the following three reading tasks: (1) MT battery (Cornoldi \& Colpo, 1998); (2) word reading task (Sartori et al., 1995); (3) pseudo-word reading task (Sartori et al., 1995).

The MT battery was used to obtain a measurement of the children's reading speed and accuracy while reading aloud age-standardised Italian prose passages (i.e., ecological-context reading). This was done by computing respectively the mean number of syllables/sec read as well as the number of errors made by the children.

The ability to read aloud was also measured using the word reading task (Sartori et al., 1995) consisting of four standardised clinical lists of 112 Italian words. 
Furthermore, the phonological decoding ability was then measured using the pseudo-word task (Sartori et al., 1995) consisting of three standardised clinical lists of 48 Italian pseudo-words (Sartori et al., 1995). Also in these cases, both accuracy and reading speed were scored.

Dyslexic children suffering from attention deficit hyperactivity disorder (ADHD) were excluded from the experiment, to avoid interfering and confounding effects. None of the participants had been treated for any neurological or psychiatric disorder, nor were they under pharmacological treatment at the time of the experimental session.

Ten normal readers, with no reported academic difficulties, matched to the dyslexics in age [mean age $134,3 \pm 26,0$ months, $\mathrm{t}(18)=-0.852, P=0.405]$ and gender [ 4 girls; Fisher's exact test, $P=1.00$ ), served as the control group.

All children were native Italian speakers and had normal or corrected-to-normal vision. No subjects exhibited any colour deficits, as determined by Ishihara colour plates (Ishihara, 1997). All children's parents gave their informed consent to the study, in accordance with the Declaration of Helsinki.

Table 1 shows the mean and SD of age and text reading tests for the control and dyslexic groups. Controls and dyslexics were comparable in chronological age $[t(18)=-8.852$, n.s.], but were significantly different on accuracy and speed of word, pseudo-word and text reading.

\subsection{Visual stimuli}

Stimuli were designed to preferentially activate functionally separate pathways in the visual system, traditionally described as magno-cellular (M), parvocellular (P) and konio-cellular (K) streams.

Chromatic visual stimuli were equiluminant horizontal sinusoidal gratings, modulated both in luminance (Y-Bk) and chromaticity (R-G and B-Y). Stimuli were obtained by combining red and green gratings of identical contrast and luminance. Chromatic contrast patterns (red-green or blue-yellow) were obtained by superimposing (out-of-phase by $180 \mathrm{deg}$ ) red-black and green-black gratings (or blueblack and yellow-black, respectively) of identical contrast. Luminance contrast patterns (white-black) were obtained by superimposing the same gratings in-phase (Porciatti \& Sartucci 1999). Gratings were generated by a VSG/2 graphic card (Cambridge Research ${ }^{\odot}$, UK), displayed full-field on a colour monitor (Samsung Sync Master1 100DF ${ }^{\circledR}, 21$ inches) at a frame rate of $120 \mathrm{~Hz}$ and 14 bits per colour per pixel, suitably linearised by gamma correction (Porciatti \& Sartucci, 1996; Porciatti \& Sartucci, 1999).

The equiluminant point was measured by assessing contrast sensitivity with the method of ascending limits for a $1 \mathrm{c} / \mathrm{deg}$ red-green or black-yellow grating, counterphased at $15 \mathrm{~Hz}$ (Fiorentini et al., 1996; Porciatti \& Sartucci, 1999). The point of minimum sensitivity was taken as the equiluminant value for the subject. The relative luminance (r) is easily defined by the usual formula $r=\mathrm{Lum}_{\text {red }} /\left(\mathrm{Lum}_{\text {red }}+\mathrm{Lum}_{\text {green }}\right)$, where values of $r=0, r=0.5$ (equiluminant point, at maximum chromatic contrast) and $r=1.0$ respectively define G-Bk, R-G and R-Bk patterns (Mullen, 1985). The extreme values (i.e. $r=0$ and $r=1$ ) characterise gratings with a pure luminance contrast and a poor chromatic contrast.

To minimise the contribution of short-wavelength cones, for red-green stimuli the patterns were viewed through yellow filters (Kodak Wratten 16), thus attenuating wavelengths below $500 \mathrm{~nm}$.

Chromatic contrast stimuli with a transient-onset presentation and a peak spatial frequency of about 2 $\mathrm{c} / \mathrm{deg}$ (single bar width $=15$ arcmin) with a $14 \times 14$ deg field size were adopted, as previous studies have shown that larger fields introduce luminance contamination, due both to chromatic aberration and retinal inhomogeneity (Stabell \& Stabell, 1980; Porciatti \& Sartucci, 1999). Luminance contrast stimuli were employed at two different peak spatial frequencies:

Table 1

Mean (M) and standard deviation (SD) of age and reading abilities in both control and dyslexic groups

\begin{tabular}{|c|c|c|c|c|c|c|}
\hline & \multicolumn{2}{|c|}{ Controls $(\mathrm{N}=10)$} & \multicolumn{2}{|c|}{ Dyslexics $(\mathrm{N}=10)$} & \multicolumn{2}{|c|}{ Comparison } \\
\hline & M & $\mathrm{SD}$ & M & $\mathrm{SD}$ & $\mathrm{T}(18)$ & $\mathrm{P}$ \\
\hline Age (months) & 134.30 & 26.02 & 142.30 & 14.29 & -8.852 & 0.405 \\
\hline Text reading errors (number) & 2.2 & 1.55 & 9.8 & 5.23 & -4.407 & $<0.001$ \\
\hline Text reading speed (syll/sec) & 4.004 & 1.044 & 1.541 & 0.758 & 6.037 & $<0.001$ \\
\hline Word reading errors ( $Z$-score) & 0.230 & 0.400 & -1.178 & 0.851 & 4.735 & $<0.001$ \\
\hline Word reading speed (Z-score) & 0.215 & 0.379 & -1.990 & 0.874 & 7.318 & $<0.001$ \\
\hline Pseudoword reading errors (Z-score) & 0.191 & 0.317 & -1.373 & 0.917 & 5.099 & $<0.001$ \\
\hline Pseudoword reading speed (Z-score) & 0.249 & 0.441 & -1.927 & 0.643 & 8.822 & $<0.001$ \\
\hline
\end{tabular}


2c/deg (single bar width $=15$ arcmin, i.e. small bar size) and $0.5 \mathrm{c} / \mathrm{deg}$ (single bar width $=60 \mathrm{arcmin}$, i.e. large bar size). Two different contrast levels (K90\% and $\mathrm{K} 20 \%$ ) were used for both luminance contrast and chromatic contrast for red-green VEPs recordings, while only the higher contrast level (K90\%) was used for chromatic blue-yellow VEPs recordings.

The visible screen was $26 \mathrm{~cm}$ wide and $24 \mathrm{~cm}$ high and the viewing distance $100 \mathrm{~cm}$. Mean luminance was kept at $17 \mathrm{~cd} \mathrm{~m}^{-2}$ with a retinal luminance of 330 Troland when viewed through natural pupils, measured to be about $5 \mathrm{~mm}$ in all subjects.

For any other technical information see previous works on the topic by Porciatti and Sartucci (Porciatti \& Sartucci, 1996, 1999).

\subsection{Electrophysiological recordings}

Transient VEPs were recorded on-line using a BM 623 device (Biomedica Mangoni, Pisa).

The recording $\mathrm{AgCl}$ electrode was placed on the Oz position of the 10-20 International EEG System, while the reference electrode was positioned over $\mathrm{Cz}$ and the ground was located on the forehead (Harding et al., 1996; Porciatti \& Sartucci, 1999; Tobimatsu et al., 2000).

VEPs were recorded in response to abrupt reversal $(1 \mathrm{reversal} / \mathrm{sec}=1 \mathrm{~Hz})$ of a horizontal square wave grating (see above for spatial frequency and contrast features). As a consequence, the duration of each stimulus as well as the recording time-window was $500 \mathrm{~ms}$.

Subjects maintained stable fixation on a dot (diameter, $0.2^{\circ}$ ) throughout stimulus presentation. The display was centred on the vertical meridian (central stimulation). In accordance with the international recommendations for visual system testing (Holder et al., 2010), both eyes were stimulated for each participant, one eye at a time (other eye patched) in random order, in order to avoid a binocular summation of the amplitudes of evoked responses.

Fifty stimuli were delivered for each stimulus condition (block) and for each eye. Block sequences occurred in random order. The whole protocol had a duration of about $45^{\prime}$ including pauses between blocks. All participants were naïve to VEP recordings and were only admitted into the experimental room immediately before the recording session.

Signals were filtered $(0.3 \pm 100 \mathrm{~Hz}, 26 \mathrm{~dB} / \mathrm{oct})$ amplified (50 000 fold), digitised $(2 \mathrm{kHz}, 12$ bit resolution) and averaged (at least 50 sums), with a rejection of signals exceeding a threshold voltage.
Partial averages (blocks of 10 sums) of total average were used to evaluate response consistency (Porciatti \& Sartucci, 1999; Caleo et al., 2007; Bocci et al., 2014). At least two series of 50 events (total: 100 traces) were averaged with the stimulus contrast reversal.

VEPs were measured in terms of both latency from the stimulus onset (in ms) and amplitude (in $\mu \mathrm{V}$ ). The amplitude of the $\mathrm{P} 1$ component (obtained from achromatic stimuli) was measured peak-to-peak (i.e., with respect to the peak of the preceding negative wave), while the amplitude of the N1 component (obtained from chromatic stimuli) was measured baseline-topeak (i.e., with respect to the first $50 \mathrm{~ms}$ of the recording trace taken as baseline) (for ref. see Holder et al., 2010).

For any other technical information see previous works on the topic by Porciatti and Sartucci (Porciatti \& Sartucci, 1996, 1999).

\subsection{Statistical analysis}

Statistical analysis was carried out using the Statistical Package for the Social Sciences (SPSS, Inc., Chicago, IL, USA).

Differences between the two groups regarding demographic items and reading abilities were analysed by means of Student's $t$ tests (or Fisher's exact test when comparing gender proportions).

A series of two-way ANOVAs with a $2 \times 2$ factorial design (two factors and two levels for each factor) was carried out in order to assess the possible presence of main and/or interaction effects on VEPs latency and amplitude. The reading ability factor (with normal and dyslexic reading as levels) was tested from time to time with the following factors: (a) luminance contrast (with low and high contrast as levels), (b) stimulus size (with small and large bar size as levels), for black/white stimuli; (c) chromatic contrast (with low and high contrast as levels), for isoluminant red/green stimuli; (d) chromatic channel (with red/green and blue/yellow opponent channel as levels). The normality of distribution of each variable was tested and transformed data were used when necessary. Between- and within-group multiple comparisons were made by means of the Tukey post-hoc test. Data were presented as mean $\pm \mathrm{SEM}$; a level of $5 \%$ probability $(p<0.05)$ was considered significant.

Effect sizes were also computed in order to provide a measurement of the magnitude of the observed effects and, then, to aid their practical interpretation. Effect size relative to the two-way ANOVA tests 
was estimated using the partial Eta-Squared $\left(\eta^{2}\right)$. A commonly used interpretation is to refer to effect size as small $\left(\eta^{2}{ }_{p}=0.01\right)$, medium $\left(d=\eta^{2}{ }_{p}=0.06\right)$, and large $\left(\eta_{p}^{2}=0.14\right)$ based on $\eta^{2}$ penchmarks suggested by Cohen (1988). When an interaction was present, the value of the interaction coefficient was also reported. Effect size relative to the post-hoc (Tukey) tests was estimated by using the appropriate Cohen's index $(d)$. Cohen (1988) has provided $d$ benchmarks to define small $(d=0.2)$, medium $(d=0.5)$, and large $(d=0.8)$ effects. The betweengroup difference of means was also reported.

Power (1- $\beta)$ of all the performed tests with $\alpha=0.050$ were also calculated and reported. A generally accepted minimum level of power is 0.80 (Cohen, 1988).

\section{Results}

Results are summarized in Table 2, where data are reported as mean values \pm SD of VEPs latency and amplitude in response to either luminance contrast (P1 component from black/white pattern) or chromatic contrast stimuli (N1 component from red/green and blue/yellow equiluminant patterns) for both normal and dyslexic readers.

As a representative example, Fig. 1 depicts both grand average and individual VEPs traces recorded from either normal or dyslexic readers to red-green equiluminant patterns at high chromatic contrast.

\subsection{Luminance contrast}

A two-way ANOVA was performed to determine whether the reading ability and the luminance contrast of the black/white stimulus affected P1 latency (Fig. 2A). A main effect of luminance contrast was found, $\mathrm{F}(1,65)=39.232, p<0.001, \eta^{2}{ }_{\mathrm{p}}=0.38$, $1-\beta=1$, indicating that the low contrast level yielded
P1 latencies that were delayed with respect to the high one. There was also a main effect on the reading ability, $\mathrm{F}(1,65)=10.688, p<0.01, \eta_{\mathrm{p}}^{2}=0.14,1-\beta=0.89$, showing that dyslexic readers produced overall later responses than normal readers. Finally, there was an interaction between luminance contrast and reading ability, $\mathrm{F}(1,65)=6.147, p<0.05, \eta^{2} \mathrm{p}=0.09$, $1-\beta=0.69$, interaction coefficient $=10.89$, so that the contrast effect depended on what kind of reading ability was present. In particular, post-hoc tests (Tukey) showed that, at low contrast, dyslexic readers generated significantly later responses than normal readers, $p<0.001$. Furthermore, a simple effect of the luminance contrast was found at each level of reading ability, being stronger in dyslexic (difference of means $=19.2, p<0.001, d=1.27$ ) than in normal readers (difference of means $=8,32, p<0.01$, $d=0.66$ ).

The reading ability and the luminance contrast were also tested to determine whether they were able to affect P1 amplitude (Fig. 2B). A two-way ANOVA found only a main effect of luminance contrast, $\mathrm{F}(1,65)=5.951, p<0.05, \eta^{2}{ }_{\mathrm{p}}=0.08,1$ $\beta=0.67$, indicating that the low contrast level yielded P1 amplitudes that were smaller with respect to the high one. However, no simple effects were found between treatment groups.

In addition, the reading ability and the size of the black/white stimulus (set at the high contrast level) were tested to determine whether they were able to affect both P1 latency and amplitude, but no effects were found (Fig. 2C,D).

\subsection{Chromatic contrast}

A two-way ANOVA was performed to determine whether the reading ability and the chromatic contrast of the red/green stimulus affected N1 latency (Fig. 3A).

Table 2

Synoptic view of VEP latencies and amplitudes in both control and dyslexic groups

\begin{tabular}{|c|c|c|c|c|c|}
\hline \multirow[t]{2}{*}{ Stimulus type } & \multirow[t]{2}{*}{ Wave } & \multicolumn{2}{|c|}{ Latency (ms) } & \multicolumn{2}{|c|}{ Amplitude $(\mu \mathrm{V})$} \\
\hline & & Normal readers & Dyslexic readers & Normal readers & Dyslexic readers \\
\hline B/W Sz 60’ K 20\% & $\mathrm{P} 1$ & $118.96 \pm 7.6$ & $131.54 \pm 16.55$ & $10.44 \pm 6.41$ & $10.24 \pm 2.88$ \\
\hline B/W Sz 60’ K 90\% & $\mathrm{P} 1$ & $110.61 \pm 5.72$ & $112.34 \pm 6.64$ & $14.04 \pm 5.74$ & $13.69 \pm 6.1$ \\
\hline $\mathrm{B} / \mathrm{W} \mathrm{Sz} 15^{\prime} \mathrm{K} 90 \%$ & $\mathrm{P} 1$ & $112.43 \pm 7.83$ & $116.37 \pm 8.39$ & $12.75 \pm 5.17$ & $10.57 \pm 5.72$ \\
\hline R/G Sz 15’ K 20\% & N1 & $182.88 \pm 13.83$ & $199.10 \pm 29.17$ & $6.82 \pm 3.45$ & $7.07 \pm 3.17$ \\
\hline R/G Sz 15’ K 90\% & N1 & $166.77 \pm 8.11$ & $199.50 \pm 17.08$ & $10.83 \pm 4.96$ & $9.44 \pm 5.15$ \\
\hline $\mathrm{B} / \mathrm{Y} \mathrm{Sz} 15^{\prime} \mathrm{K} 90 \%$ & N1 & $180.98 \pm 12.53$ & $192.33 \pm 14.97$ & $9.01 \pm 6.25$ & $8.66 \pm 3.78$ \\
\hline
\end{tabular}

Data are reported as mean values $\pm \mathrm{SD}$. B/W=Black/White; R/G=Red/Green; B/Y = Blue/Yellow. Sz=stimulus size (in arcminutes); $\mathrm{K}=$ stimulus contrast. 


\section{RED/GREEN, K $90 \%$}

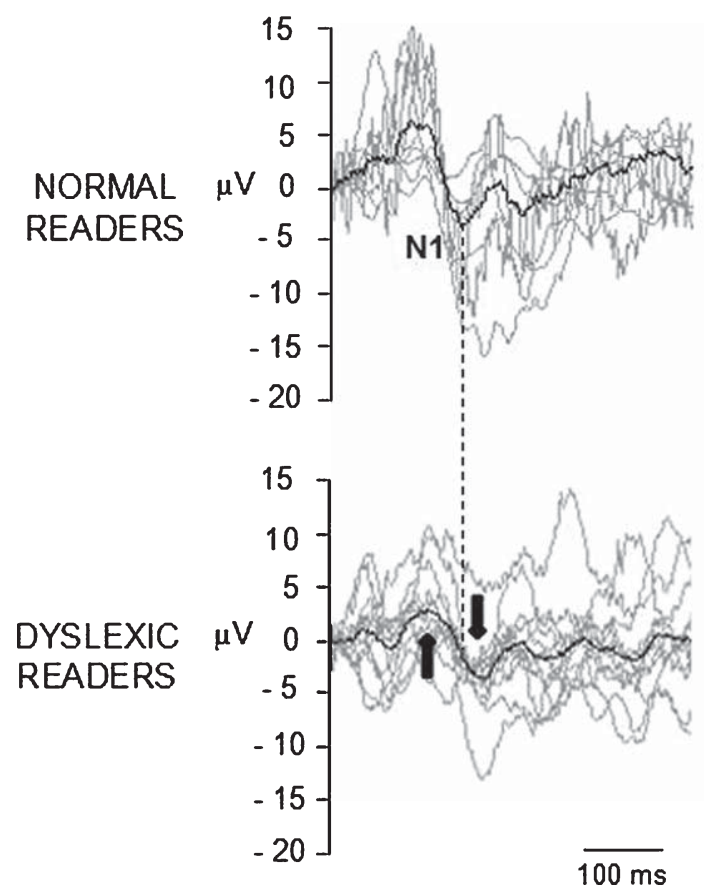

Fig. 1. VEPs recorded in response to red/green high contrast sinusoidal gratings. Note the reduced amplitude (even if not statistically significant, black arrows) and the delayed latency (dotted lines) of dyslexic readers (lower panel) compared to normal readers (upper panel). switching from low to high chromatic contrast the dyslexic readers behaviour diverges from that of normal readers, since the N1 latency does not decrease, but rather remains unchanged (Fig. 3A). In other words, increasing chromatic contrast in dyslexic readers does not mean improving the visual perception of the stimulus.

This result assumes even more significance if we consider that red/green high contrast stimuli yielded $\mathrm{N} 1$ latencies that were greater than $2 \mathrm{SD}$ above the mean of controls in 16 out of 20 eyes tested (i. e., in $80 \%$ of eyes). That is to say that N1 latencies were abnormal in all 10 subjects examined in at least one eye (i. e., in $100 \%$ of subjects).

Furthermore, a simple effect of chromatic contrast was found in normal readers (difference of means $=16,11, p<0.05$ ), but not in dyslexic readers.

The reading ability and the chromatic contrast were also tested to determine whether they were able to affect N1 amplitude (Fig. 3B). A two-way ANOVA found only a main effect of chromatic contrast, $F(1,59)=7.234, p<0.01, \eta_{p}^{2}=0.11,1$ $\beta=0.76$, indicating that the low contrast level yielded $\mathrm{N} 1$ amplitudes that were smaller with respect to the high contrast level.

\subsection{Chromatic systems}

A two-way ANOVA was performed to determine whether the reading ability and the kind of chromatic stimulus delivered (red/green or blue/yellow), and thus the kind of chromatic system involved (parvocellular or koniocellular, respectively), affected N1 latency (Fig. 4A).

A main effect of reading ability was found, $\mathrm{F}(1,67)=43.830, p<0.001, \eta_{p}^{2}=0.39,1-\beta=1$, indicating that dyslexic readers produced overall later N1 responses than normal readers. Moreover, there was an interaction between the reading ability and the kind of chromatic stimulus, $F(1,67)=10.316$, $p<0.01, \eta^{2}=0.14,1-\beta=0.89$, interaction coefficient $=21.38$, so that the reading ability effect depended on the kind of chromatic stimulus delivered. In particular, post-hoc tests (Tukey) showed that dyslexic readers generated significantly later N1 responses than normal readers with both red/green stimuli (difference of means $=32.729, p<0.001$, $d=1.73$ ) and blue/yellow stimuli (difference of means $=11.346, p<0.05, d=0.57)$. The so-called difference of differences (32.729 - 11.346), which corresponds to the interaction coefficient (21.38), accounts for the N1 latency antithetical behaviour between dyslexic and normal readers $(t=3.212, p<0.01)$ 

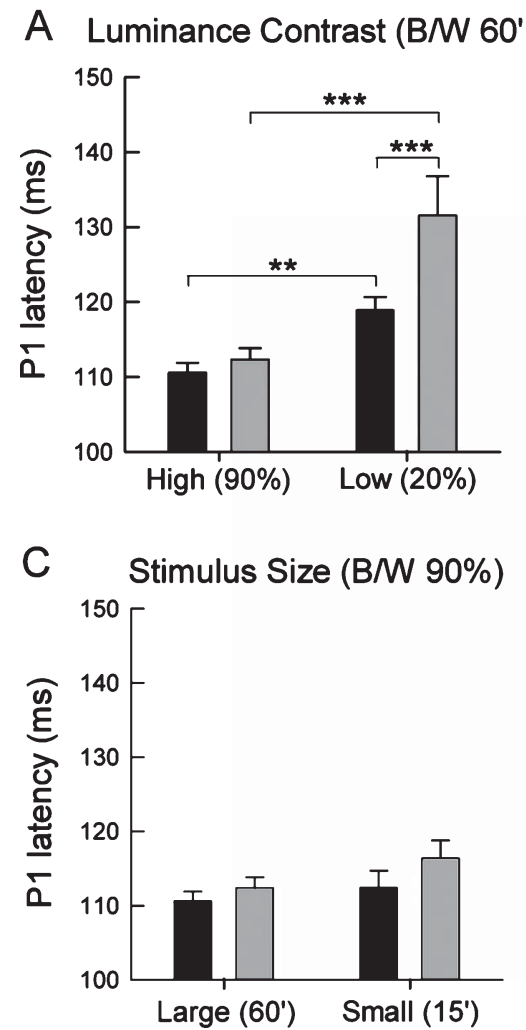
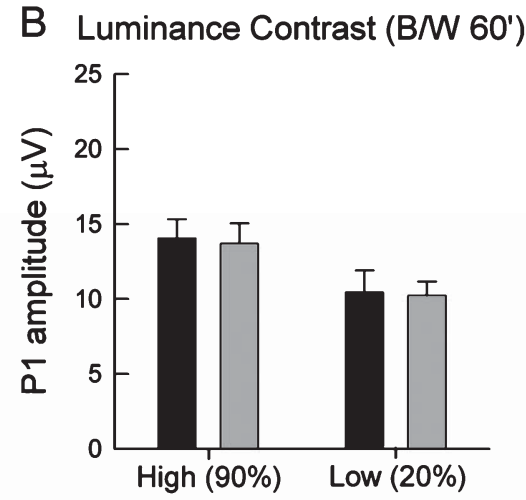

D Stimulus Size (BN 90\%)

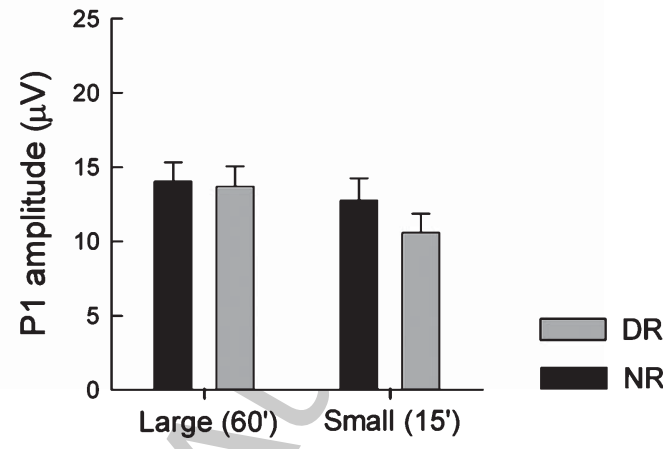

Fig. 2. Effects of luminance contrast and reading ability on P1 latency (panel 1A) and P1 amplitude (panel 1B). Effects of stimulus size (by setting luminance at high contrast) and reading ability on P1 latency (panel 1C) and P1 amplitude (panel 1D). Each bar represents the corresponding mean value \pm SEM. The different fill colours of the bars represent the two levels of reading ability: black indicates Normal Readers (NR) and grey Dyslexic Readers (DR). Data were analysed by two-way ANOVA and Tukey post-hoc test as described in Methods. ${ }^{* * *}<0.001 ;{ }^{* *}<0.01 ;{ }^{*}<0.05$. See Results for Main effects and Interactions.
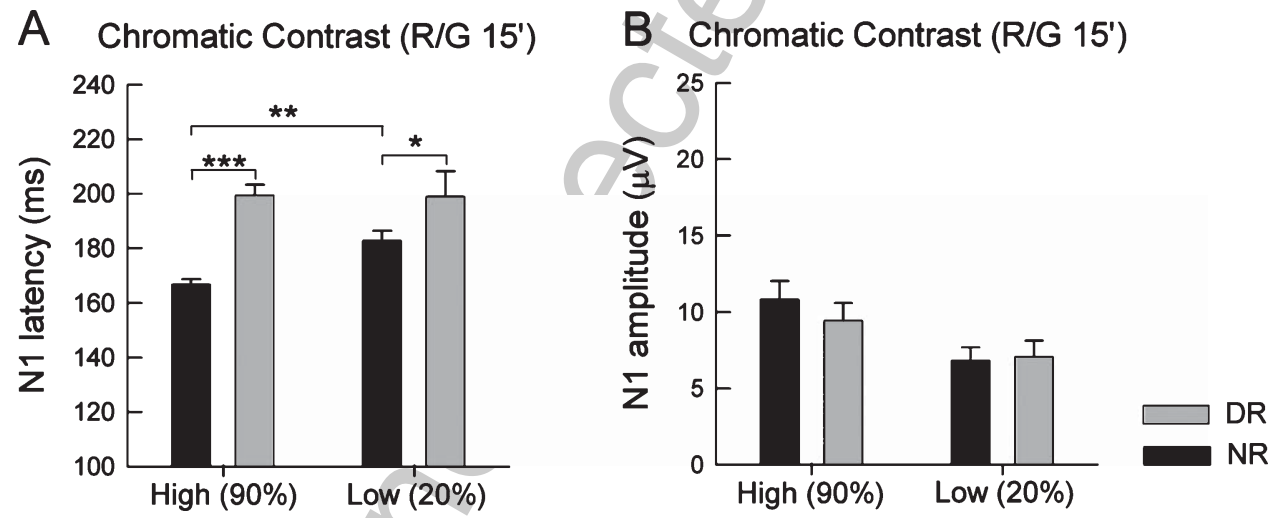

Fig. 3. Effects of chromatic contrast and reading ability on N1 latency (panel A) and N1 amplitude (panel B). Each bar represents the mean value \pm SEM. Fill colours of the bars as in Fig. 2. Data were analysed by two-way ANOVA and Tukey post-hoc test as described in Methods. $* * *<0.001 ;{ }^{* *}<0.01 ; *<0.05$. See Results for Main effects.

when switching from blue/yellow to red/green stimuli (e.g., in dyslexic readers the N1 latency does not decrease, as in normal readers, but rather increases) (Fig. 4A).
Furthermore, a simple effect of the kind of chromatic stimulus delivered was found in normal readers (difference of means $=14.208, p<0.01$ ), but not in dyslexic readers. 
A Chromatic channels (15' $90 \%$ )

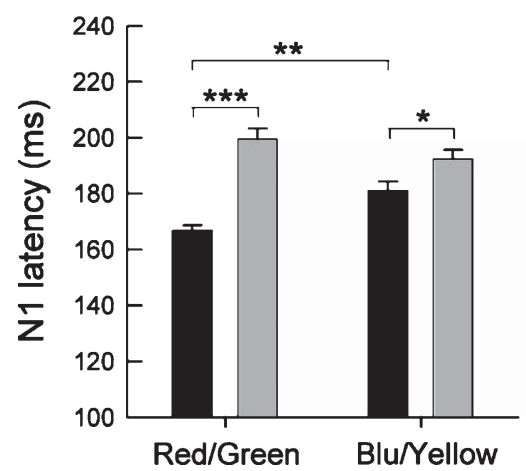

B Chromatic Channels (15' 90\%)

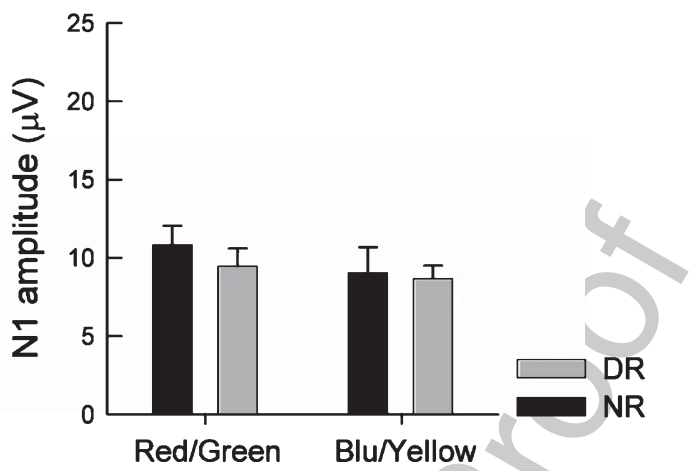

Fig. 4. Effects of chromatic channel and reading ability on N1 latency (panel A) and N1 amplitude (panel B). Each bar represents the mean value \pm SEM. Fill colours of the bars as in Fig. 2. Data were analysed by two-way ANOVA and Tukey post-hoc test as described in Methods. ${ }^{* * *}<0.001 ;{ }^{* *}<0.01 ;{ }^{*}<0.05$. See Results for Main effects and Interactions.

The reading ability and the kind of chromatic stimulus delivered were also tested to determine whether they were able to affect $\mathrm{N} 1$ amplitude, but no effects were found (Fig. 4B).

\section{Discussion}

\subsection{General findings}

At group level, dyslexic children showed delayed evoked responses to both achromatic stimuli (magnocellular-dorsal stream) and isoluminant red/green chromatic stimuli (parvocellular-ventral stream) compared with age-matched normal readers. Notably, dyslexic children also developed altered responses to isoluminant blue/yellow chromatic stimuli (koniocellular system). To our knowledge, this is the first time that a dysfunction of colour vision has been brought to light in an objective way (i.e., by means of electrophysiological methods) in children with DD.

Our results concerning poor perceptual sensitivity of dyslexic children towards achromatic stimuli with low spatial frequency and low luminance contrast confirm those obtained by others (May et al., 1991; Maddock et al., 1992; Romani et al., 2001; Samar et al., 2002; Vaegan \& Hollows, 2006) supporting the hypothesis of a magnocellular-dorsal weakness as an integral part of the neurobiological substrate of developmental dyslexia. This is a hypothesis that still maintains its objective value, even though it has been reformulated with respect to the original magnocellular theory and is now known as "sluggish attentional shifting (SAS) hypothesis" (Hari \&
Renvall, 2001). The magnocellular system dysfunction, in fact, is now placed in relation with a deficit of the (visual) attentional shifting. This is crucial, in its spatial and temporal aspects, in the parsing of sublexical orthographic units (graphemic parsing) (Gori \& Facoetti, 2014). This latter aspect is necessary for both individual letter processing and letter-tospeech sound integration (Vidyasagar \& Pammer, 2010).

Our results concerning the poor perceptual sensitivity of dyslexic children to isoluminant red/green chromatic stimuli with high chromatic contrast and high spatial frequency, strengthen and corroborate the hypothesis of an involvement of the parvocellular system firstly proposed by Farrag and colleagues (Farrag et al., 2002). In this work, delayed electrophysiological responses to achromatic stimuli with high spatial frequency were found in dyslexic children with respect to normal readers. Nevertheless, the fact that the stimuli used were achromatic, and as such not entirely selective for the parvocellular system, could leave the doubt that response delays depended to a certain extent on the co-activation of the magnocellular system. More recently, however, the parvocellular hypothesis has received a new impetus since, by employing colour stimuli (thus selective for the parvocellular system), higher red-green isoluminant points were shown in dyslexic children compared to controls (Ahmadi et al., 2015). Our results, obtained by means of an electrophysiological (objective) method, converge with those of Ahamadi and colleagues (Ahamdi et al., 2015), obtained by means of a psychophysiological (subjective) method. It follows that the reliability of both results is mutually reinforced. 
Finally, the involvement we have found of the koniocellular system may be related to both of the following: a low sensitivity to opponent blue/yellow chromatic stimuli, supporting the notion of a visual impairment that extends to the entire chromatic axis; a poor perceptual sensitivity to motion, given the known contribution of the koniocellular system to the magnocellular-dorsal stream (Shipp, 2006).

\subsection{Colour vision in developmental dyslexia: A brief historical review}

A growing body of literature exists about colour vision in developmental dyslexia, although it primarily focuses on several empirical findings regarding the possibility of obtaining an improvement in reading by means of colour lenses or colour filters (see Irlen, 1991), while the underlying neurobiological substrate has not been sufficiently explored. Ray and colleagues (Ray et al., 2005) showed that wearing yellow filters for a period of three months may improve the reading abilities in dyslexic children. This booster effect seems to emerge through the removal of the S-cone inhibitory input on the magnocellular system. Yellow filters, in fact, seem to be able to cut off short wavelengths from the visible light spectrum (the socalled 'negative blue') and, in addition, to increase the phase coherence of the L- (red-) and M- (green-) cone input, thus normalising the cone contrast weighting. All this seems to result, on the whole, in an increase in efficiency of the magnocellular system (Ray et al., 2005). It should be noted, however, that these measures, changing the background colour with respect to the text, do not change the chromatic contrast but rather the luminance contrast (non-opposing achromatic channel) (Kremers \& Link, 2008).

In addition, a study by Dain and colleagues (Dain et al., 2008), conducted with psychophysical (i.e., subjective) methods, revealed lower perceptual thresholds to yellow/blue stimuli in dyslexic children compared with controls, suggesting a dysfunction in colour vision in dyslexic children (dysfunctional hypersensitivity) restricted to the koniocellular system.

Finally, although further evidence of a colour vision dysfunction has been widely sought in dyslexic children, even in recent years (Gori et al., 2014), it has never been found before now, perhaps due to the inappropriateness of the visual stimuli used. On the contrary, both in the previously mentioned work of Ahmadi and colleagues (Ahmadi et al., 2015) and in the present study, red/green and blue/yellow isoluminant stimuli, which are highly selective for either the parvocellular or the koniocellular pathways, were employed.

\subsection{Hypotheses regarding parvocellular system involvement}

Bearing in mind the specific functions that are classically attributed to each system, namely, for the magnocellular-dorsal system, the visual attention upon object spatial characteristics (visual localization) and, for the parvocellular-ventral system, the visual attention upon object specific features (visual recognition), a dysfunction of both systems would well explain impairments of both reading progression and recognition of letter details that characterise reading disorders in dyslexics.

Moreover, the demonstration of an impairment of both systems would provide new elements to the hypothesis of a modulatory influence of the magnocellular system towards the parvocellular system. In fact, it is known that magnocellular inputs reach the primary visual cortex earlier than parvocellular ones (Laycock et al., 2008). This temporal advantage, according to recent studies, would give the opportunity to the magnocellular system to exert a top-down facilitatory control on the parvocellular system through a reentrant loop of projection (upon the ventral system), via the orbitofrontal cortex and the fusiform gyrus (Kveraga et al., 2007; Tapia \& Breitmeyer, 2011). Furthermore, considering that the magnocellular system reaches full development at the age of 2-3 months (Crognale, 2002), while the parvocellular one much later, at the turn of adolescence (Crognale, 2002; Pompe et al., 2006), one could assume that in the presence of an incomplete development of the magnocellular system the parvocellular system also suffers a delayed maturation.

\subsection{Possible cues for (re)habilitation}

The fact that both systems can contribute to the biological substrate of DD induces some speculation concerning activities to be promoted, and/or exercises to be undertaken, in real life environments in order to prevent and/or improve reading disorders in these children. A wellness program for reading abilities designed on the basis of what has emerged from this study should aim, in our opinion, toward the following two main objectives: a) to enhance the magnocellular-dorsal system function and, at the 
same time, b) to support the parvocellular-ventral system function and its development.

\subsubsection{Magnocellular-dorsal system}

As far as the magnocellular-dorsal system is concerned, its main function is to direct visuospatial attention and, consequently, to control the sequence of saccades for reading progression (Iles et al., 2000; Seassau et al., 2014). It has been shown recently that playing action video games (AVG), which implies dealing with quickly and unpredictably moving objects in the peripheral visual field, immediately improves reading skills of dyslexic children, probably by improving visual navigation skills (Franceschini et al., 2013). As a consequence, (motion) perceptual learning and AVG has been proposed by Gori and Facoetti for rehabilitation and/or educational purposes (Gori e Facoetti, 2014; see also Karimpur \& Hamburger, 2015).

From the same perspective, in our opinion, proposing figure-ground perception games (such as hidden pictures or Where's Waldo) could have the same effect of favouring scanning abilities that are crucial for the successful acquisition of reading abilities. Moreover, since the magnocellular-dorsal system is deemed to be a perception-and-action system able to mediate visually guided behaviour (such as reaching, grasping and self-locomotion) (see Ashley, 2004), in our opinion, children with or at risk of DD could also benefit from practicing sports and games that train such skills (for instance the so-called ball sports, such as racquet-and-ball or net goal sports).

\subsubsection{Parvocellular-ventral system}

As far as the parvocellular-ventral system is concerned, its main function is to discriminate and recognise shapes and objects. It has been shown recently that exercise in free-form printing of manuscript letters triggers a writing-reading network that includes both fronto-parietal regions (involved in writing) and the visual word form area (part of the ventral system and involved in reading and letter processing). This would facilitate reading acquisition through an improved effectiveness in recruiting the left fusiform gyrus during reading performance alone (James \& Engelhardt, 2012). As a consequence, handwriting exercises have also been proposed by James and Engelhardt (2012) so that children can learn to recognise those attributes of letters (such as shape and orientation) which are relevant for their successful identification and categorisation.
Similarly, in our opinion, proposing puzzle games (like Tetris or Tangram) could boost discrimination and recognition abilities of shape, size and orientation of geometric figures, which underlie individual letter identification.

\section{Concluding remarks}

In conclusion, the group of dyslexic children showed delayed evoked responses of all the visual pathways examined and, in particular, a complete involvement of the visual chromatic axis. The amplitude of the evoked responses, on the contrary, was not significantly reduced compared to normal readers. This dissociation suggests a general slowing of visual processing as a key feature of DD, consistent with a delayed myelination (i.e., dysmaturation) rather than with a reduced number of axons/neurons (Walsh et al., 2005; American Clinical Neurophysiology Society, 2006). This agrees with previous diffusion tensor imaging studies that detected in dyslexic brains abnormalities of fractional anisotropy and radial diffusivity consistent with disrupted myelination in the left superior longitudinal (arcuate) fasciculus (Deutsch et al., 2005; Vandermosten et al., 2012). However, the results obtained in the present study suggest that dysmyelination might be a widespread phenomenon in dyslexic brains, extending even outside the limits of the language network.

Our results do not necessarily imply that achromatic and chromatic visual impairments have to be considered the cause of DD. In fact, they could simply represent an effect of DD or the product of third factors. However, they can certainly be considered as part of the neurobiological substrate of DD.

These results have led us to make some considerations, no more than speculative at the present stage of our research, concerning a putative wellness program that could aim to promote learning how to read and/or improve existing reading skills of children with, or at risk of DD. The intention is to introduce enhancing interactions and/or additive or combined effects, able to support the development of both visual systems as well as learning to read. This would occur through the induction of synaptogenesis and myelination, in a period of life in which brain plasticity is at its maximum (Kolb \& Gibb, 2014).

Obviously, the effectiveness of our working hypothesis will necessarily have to be tested by means of specially designed studies and sufficiently large numbers of patients before it can become part of 
an accredited wellness program of reading prerequisites (i.e., a program aimed at the development and/or reinforcement of reading prerequisites); a wellness programme that is not to be considered as an alternative to the classic auditory-phonological approach but rather could usefully be associated to it.

\section{Acknowledgments}

The authors wish to thank Mr. Carlo Orsini for his excellent technical assistance and Prof. Fiona Meert for language revision.

\section{References}

Ahmadi, K., Pouretemad, H.R., Esfandiari, J., Yoonessi, A., \& Yoonessi, A. (2015). Psychophysical evidence for impaired magno, parvo, and konio-cellular pathways in dyslexic children. Journal of Ophthalmic and Vision Research, 10(4), 433-440.

American Clinical Neurophysiology Society (2006). Guideline 9B: Guidelines on visual evoked potentials. American Journal of Electroneurodiagnostic Technology, 46(3), 254-274.

Ashley, M.J. (2004). Traumatic brain injury: Rehabilitative treatment and case mangement. Boca Raton, FL: CRC Press.

Bocci, T., Caleo, M., Tognazzi, S., Francini, N., Briscese, L., Maffei, L., Rossi, S., Priori, A., \& Sartucci, F. (2014). Evidence for metaplasticity in the human visual cortex. Journal of Neural Transmission, 121(3), 221-231.

Born, R.T., \& Bradley, D.C. (2005). Structure and function of visual area MT. Annual Review of Neuroscience, 28, 157-189.

Brown, J.M. (2009). Visual streams and shifting attention. Progress in Brain Research, 176, 47-63.

Burr, D.C., Morrone, M.C., \& Ross, J. (1994). Selective suppression of the magnocellular visual pathway during saccadic eye movements. Nature, 371(6497), 511-513.

Caleo, M., Restani, L., Gianfranceschi, L., Costantini, L., Rossi, C., Rossetto, O., Montecucco, C., \& Maffei, L. (2007). Transient synaptic silencing of developing striate cortex has persistent effects on visual function and plasticity. The Journal of Neuroscience, 27(17), 4530-4540.

Chatterjee, S., \& Callaway, E.M. (2002). S cone contributions to the magnocellular visual pathway in macaque monkey. Neuron, 35(6), 1135-1146.

Cohen, J. (1988). Statistical power analysis for the behavioral sciences. New York, NY: Routledge Academic.

Cornoldi, C., \& Colpo, M. (1998). Prove di lettura MT per la scuola elementare-2 [Italian MT reading tests for primary school-2]. Firenze, Italy: Organizzazioni speciali.

Crognale, M.A. (2002). Development, maturation, and aging of chromatic visual pathways: VEP results. Journal of Vision, 2, 438-450.
Dain, S.J., Floyd, R.A., \& Elliot, R.T. (2008). Color and luminance increment thresholds in poor readers. Visual Neuroscience, 25(3), 481-486.

Deutsch, G.K., Dougherty, R.F., Bammer, R., Siok, W.T., Gabrieli, J.D., \& Wandell, B. (2005). Children's reading performance is correlated with white matter structure measured by diffusion tensor imaging. Cortex, 41(3), 354-363.

Farrag, A.F., Khedr, E.M., \& Abel-Naser, W. (2002). Impaired parvocellular pathway in dyslexic children. European Journal of Neurology, 9(4), 359-363.

Fiorentini, A., Porciatti, V., Morrone, M.C., \& Burr, D.C. (1996). Visual ageing: Unspecific decline of the responses to luminance and colour. Vision Research, 36(21), 3557-3566.

Franceschini, S., Gori, S., Ruffino, M., Viola, S., Molteni, M., \& Facoetti, A. (2013). Action video games make dyslexic children read better. Current Biology, 23, 462-466.

Galaburda, A., \& Livingstone, M. (1993). Evidence for a magnocellular defect in developmental dyslexia. Annals of the New York Academy of Sciences, 682, 70-82.

Gori, S., \& Facoetti, A. (2014). Perceptual learning as a possible new approach for remediation and prevention of developmental dyslexia. Vision Research, 99, 78-87.

Gori, S., Cecchini, P., Bigoni, A., Molteni, M., \& Facoetti, A. (2014). Magnocellular-dorsal pathway and sub-lexical route in developmental dyslexia. Frontiers in Human Neuroscience, $8,460$.

Gori, S., Mascheretti, S., Giora, E., Ronconi, L., Ruffino, M., Quadrelli, E., Facoetti, A., \& Marino, C. (2015). The DCDC2 intron 2 deletion impairs illusory motion perception unveiling the selective role of magnocellular-dorsal stream in reading (dis)ability. Cerebral Cortex, 25(6), 1685-1695.

Harding, G.F., Odom, J.V., Spileers, W., \& Spekreijse, H. (1996). Standard for visual evoked potentials 1995. The International Society for Clinical Electrophysiology of Vision. Vision Research, 36(21), 3567-3572.

Hari, R., \& Renvall, H. (2001). Impaired processing of rapid stimulus sequences in dyslexia. Trends in Cognitive Sciences, 5(12), 525-532.

Heywood, C.A., Gadotti, A., \& Cowey, A. (1992). Cortical area V4 and its role in the perception of color. Journal of Neuroscience, 12(10), 4056-4065.

Holder, G.E., Celesia, G.G., Miyake, Y., Tobimatsu, S., \& Weleber, R.G. (2010). International federation of Clinical Neurophysiology: Recommendations for visual system testing. Clinical Neurophysiology, 121(9), 1393-1409.

Hornickel, J., \& Kraus, N. (2013). Unstable representation of sound: A biological marker of dyslexia. The Journal of Neuroscience, 33(8), 3500-3504.

Iles, J., Walsh, V., \& Richardson, A. (2000). Visual search performance in dyslexia. Dyslexia, 6(3), 163-177.

Irlen, H. (1991). Reading by the colors: Overcoming dyslexia and other reading disabilities through the Irlen method. New York, NY: Avery Publishing Group.

Ishihara, S. (1997). Tests for colour-deficiency. Tokyo, Japan: Kanehara.

James, K.H., \& Engelhardt, L. (2012). The effects of handwriting experience on functional brain development in pre-literate children. Trends in Neuroscience and Education, 1(1), 32-42. 
Karimpur, H., \& Hamburger, K. (2015). The future of action video games in psychological research and application. Frontiers in Psychology, 6, 1747.

Kolb, B., \& Gibb, R. (2014). Searching for the principles of brain plasticity and behavior. Cortex, 58, 251-260.

Kremers, J., \& Link, B. (2008). Electroretinographic responses that may reflect activity of parvo- and magnocellular postreceptoral visual pathways. Journal of Vision, 8(15), 11-14.

Kuba, M., Szanyi, J., Gayer, D., Kremlácek, J., \& Kubová, Z. (2001). Electrophysiological testing of dyslexia. Acta Medica (Hradec Kralove), 44(4), 131-134.

Kubová, Z., Kuba, M., Peregrin, J., \& Nováková, V. (1996). Visual evoked potential evidence for magnocellular system deficit in dyslexia. Physiological Research/Academia Scientiarum Bohemoslovaca, 45(1), 87-89.

Kveraga, K., Boshyan, J., \& Bar, M. (2007). Magnocellular projections as the trigger of top-down facilitation in recognition. The Journal of Neuroscience, 27(48), 13232-13240.

Laycock, R., Crewther, D.P., \& Crewther, S.G. (2008). The advantage in being magnocellular: A few more remarks on attention and the magnocellular system. Neuroscience \& Biobehavioral Reviews, 32(8), 1409-1415.

Livingstone, M.S., Rosen, G.D., Drislane, F.W., \& Galaburda, A.M. (1991). Physiological and anatomical evidence for a magnocellular defect in developmental dyslexia. Proceedings of the National Academy of Sciences of the United States of America, 88(18), 7943-7947.

Lovegrove, W., Martin, F., Bowling, A., Blackwood, M., Badcock, D., \& Paxton, S. (1982). Contrast sensitivity functions and specific reading disability. Neuropsychologia, 20(3), 309-315.

Lyon, G.R., Shaywitz, S.E., \& Shaywitz, B.A. (2003). A definition of dyslexia. Annals of Dyslexia, 53(1), 1-14.

Maddock, H., Richardson, A.J., \& Stein, J.F. (1992). Reduced and delayed visual evoked potentials in dyslexics. Journal of Physiology, 459, 130P.

May, J.G., Lovegrove, W.J., Martin, F., \& Nelson, P. (1991). Pattern-elicited visual evoked potentials in good and poor readers. Clinical Vision Sciences, 6, 131-136.

Mullen, K.T. (1985). The contrast sensitivity of human colour vision to red-green and blue-yellow chromatic gratings. The Journal of Physiology, 359, 381-400.

Olulade, O.A., Napoliello, E.M., \& Eden, G.F. (2013). Abnormal visual motion processing is not a cause of dyslexia. Neuron, 79(1), 180-190.

Orsini, A., \& Picone, L. (2006). WISC-III. Contributo alla taratura italiana. [WISC-III. Contribution to the Italian calibration]. Firenze, Italy: Giunti O.S. Organizzazioni Speciali.

Paulesu, E., Danelli, L., \& Berlingeri, M. (2014). Reading the dyslexic brain: Multiple dysfunctional routes revealed by a new meta-analysis of PET and fMRI activation studies. Frontiers in Human Neuroscience, 8, 830.

Peterson, R.L., \& Pennington, B.F. (2012). Seminar: Developmental dyslexia. Lancet, 379(9830), 1997-2007.

Pompe, M.T., Kranjc, B.S., \& Brecelj, J. (2006). Visual evoked potentials to red-green stimulation in schoolchildren. Visual Neuroscience, 23(3-4), 447-451.
Porciatti, V., \& Sartucci, F. (1996). Retinal and cortical evoked responses to chromatic contrast stimuli. Specific losses in both eyes of patients with multiple sclerosis and unilateral optic neuritis. Brain, 119(Pt 3), 723-740.

Porciatti, V., \& Sartucci, F. (1999). Normative data for onset VEPs to red-green and blue-yellow chromatic contrast. Clinical Neurophysiology, 110(4), 772-781.

Ray, N.J., Fowler, S., \& Stein, J.F. (2005). Yellow filters can improve magnocellular function: Motion sensitivity, convergence, accommodation, and reading. Annals of the New York Academy of Sciences, 1039, 283-293.

Ribeiro, M.J., \& Castelo-Branco, M. (2010). Psychophysical channels and ERP population responses in human visual cortex: Area summation across chromatic and achromatic pathways. Vision Research, 50(13), 1283-1291.

Romani, A., Conte, S., Callieco, R., Bergamaschi, R., Versino, M., Lanzi, G., Zambrino, C.A., \& Cosi, V. (2001). Visual evoked potential abnormalities in dyslexic children. Functional Neurology, 16(3), 219-229.

Samar, V.J., Parasnis, I., \& Berent, G.P. (2002). Deaf poor readers' pattern reversal visual evoked potentials suggest magnocellular system deficits: Implication for diagnostic neuroimaging of dyslexia in deaf individuals. Brain and Language, 80(1), 21-44.

Sartori, G., Job, R., \& Tressoldi, P.E. (1995). Batteria per la valutazione della dislessia e della disortografia evolutiva [Battery for the assessment of developmental dyslexia and dysorthography]. Firenze, Italy: Giunti O.S. Organizzazioni Speciali.

Schulte-Körne, G., Bartling, J., Deimel, W., \& Remschmidt, H. (2004). Motion-onset VEPs in dyslexia. Evidence for visual perceptual deficit. Neuroreport, 15(6), 1075-1078.

Seassau, M., Gárard, C.L., Bui-Quoc, E., \& Bucci, M.P. (2014). Binocular saccade coordination in reading and visual search: A developmental study in typical reader and dyslexic children. Frontiers in Integrative Neuroscience, 8, 85 .

Shipp, S.D. (2006). Parallel visual pathways. Advances in Clinical Neuroscience \& Rehabilitation, 6(1), 21-23.

Stabell, U., \& Stabell, B. (1980). Variation in density of macular pigmentation and in short-wave cone sensitivity with eccentricity. Journal of the Optical Society of America, 70(6), 706-711.

Sumner, P., Anderson, E.J., Sylvester, R., Haynes, J.D., \& Rees, G. (2008). Combined orientation and colour information in human V1 for both L-M and S-cone chromatic axes. Neuroimage, 39(2), 814-824.

Tapia, E., \& Breitmeyer, B.G. (2011). Visual consciousness revisited: Magnocellular and parvocellular contributions to conscious and nonconscious vision. Psychological Science, 22(7), 934-942.

Tobimatsu, S., \& Celesia, G.G. (2006). Studies of human visual pathophysiology with visual evoked potentials. Clinical Neurophysiology, 117(7), 1414-1433.

Tobimatsu, S., Celesia, G.G., Haug, B.A., Onofrj, M., Sartucci, F., \& Porciatti, V. (2000). Recent advances in clinical neurophysiology of vision. Supplements to Clinical Neurophysiology, $53,312-322$.

Vaegan, \& Hollows, F.C. (2006). Visual-evoked response, pattern electroretinogram, and psychophysical magnocellular thresh- 
olds in glaucoma, optic atrophy, and dyslexia. Optometry \& Vision Science, 83(7), 486-498.

Vandermosten, M., Boets, B., Poelmans, H., Sunaert, S., Wouters, J., \& Ghesquière, P. (2012). A tractography study in dyslexia: Neuroanatomic correlates of orthographic, phonological and speech processing. Brain, 135(Pt 3), 935-948.

Victor, J.D., Purpura, K.P., \& Conte, M.M. (1998). Chromatic and luminance interactions in spatial contrast signals. Visual Neuroscience, 15(4), 607-624.

Vidyasagar, T.R. (1999). A neuronal model of attentional spotlight: Parietal guiding the temporal. Brain Research Brain Research Reviews, 30(1), 66-76.
Vidyasagar, T.R., \& Pammer, K. (2010). Dyslexia: A deficit in visuo-spatial attention, not in phonological processing. Trends in Cognitive Sciences, 14(2), 57-63.

VV.AA. (2009). Disturbi evolutivi specifici di apprendimento. Raccomandazioni per la pratica clinica dei disturbi evolutivi specifici di apprendimento: Dislessia, disortografia, disgrafia e discalculia. Promosso da Associazione Italiana Dislessia. [Learning disabilities: Recommendations for clinical practice on dyslexia, dysorthographia, and dyscalculia]. Trento, Italy: Erickson.

Walsh, P., Kane, N., \& Butler, S. (2005). The clinical role of evoked potentials. Journal of Neurology, Neurosurgery, and Psychiatry, 76(Suppl 2), ii16-ii22. 